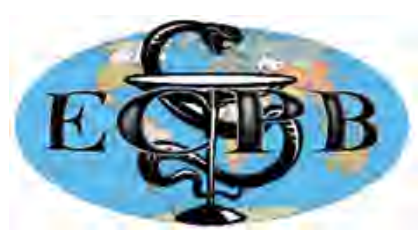

“ЕКСПЕРИМЕНТАЛЬНА ТА КЛІНІЧНА ФІЗІОЛОГІЯ І БІОХІМІЯ” "EXPERIMENTAL AND CLINICAL PHYSIOLOGY AND BIOCHEMISTRY" Науково-практичний журнал/Scientific-practical journal

Наукові статті / Research article ECPB 2018, 3(83): 5-17. https://doi.org/

\title{
Sexual Dimorphism of the Neuroendocrine-Immune Complex and its Reactions to Chronic Stress in Rats
}

\author{
I.L. POPOVYCH ${ }^{1}$, I.S. POLOVYNKO ${ }^{2}$, L.M. ZAJATS ${ }^{2}$, O.I. MELNYK ${ }^{3}$ \\ ${ }^{1}$ Department of Immunophysiology, O. Bohomolets Institute of Physiology, Kyiv, Ukraine \\ ${ }^{2}$ Department of Pathophysiology, Ivano-Frankivsk National Medical University, \\ Ivano-Frankivsk, Ukraine
}

${ }^{3}$ Department of Physiology, Danylo Halytskyi Lviv National Medical University, Lviv, Ukraine

\begin{abstract}
E-mail: i.popovych@biph.kiev.ua
E-mail: patfisiology@ifnmu.edu.ua
\end{abstract}

In our previous publications [11-18], the effect of chronic stress on immunity parameters and related changes in its neuroendocrine regulation in male and female rats were analyzed. Even a superficial cursory analysis of the data presented gives the impression of the presence of sexual differences between the parameters of the neuroendocrine-immune complex in both intact and stressed rats.

The purpose of this article is a detailed analysis of sexual dimorphism. The research is conducted in line with the concept of the neuroendocrine-immune complex [19-24].

Material and methods. The experiment was carried out on 60 female and 50 male white rats of Wistar line weighing 230-300 grams. 10 animals of each sex were not subjected to any influences while others within 7 days were under moderate stress by daily 30 -minute immobilization. The day after being stressed samples of peripheral blood (through a cut tail) were taken to analyze leukocytogram in intact and stressed rats. An hour under light ether anesthesia for 15-20 sec ECG was recorded in standard lead II (introducing needle electrodes subcutaneously) to determine parameters of heart rate variability (HRV) [1]. Then the rats were placed in individual chambers with perforated bottom to collect daily urine, in which the concentration of 17-ketosteroids was determined (by method of colorimetric reaction with m-dinitrobenzene), as well as calcium (by reaction with arsenase III), phosphates (phosphate-molybdate method), sodium and potassium (by flamming photometry).

The next day the animals were decapitated for the purpose of collecting blood. The plasma levels of the hormones of adaptation were determined: corticosterone, triiodothyronine, thyroxine and testosterone (according to ELISA) as well as electrolytes: calcium, phosphates, sodium and potassium.

According to the parameters of electrolyte exchange, hormonal activity was evaluated: parathyrin by coefficients $(\mathrm{Cap} / \mathrm{Pp})^{0,5}$ and $(\mathrm{Cap} \cdot \mathrm{Pu} / \mathrm{Pp} \cdot \mathrm{Cau})^{0,25}$, calcitonin by coefficients $(1 / \mathrm{Cap} \cdot \mathrm{Pp})^{0,25}$ and $(\mathrm{Cau} \cdot \mathrm{Pu} / \mathrm{Cap} \cdot \mathrm{Pp})^{0,25}$ as well as mineralocorticoid by coefficients $(\mathrm{Nap} / \mathrm{Kp})^{0,5}$ and $(\mathrm{Nap} \cdot \mathrm{Ku} / \mathrm{Kp} \cdot \mathrm{Nau})^{0,25}$, based on their classical effects and recommendations by I.L. Popovych $[8,19]$.

The analysis was carried out according to the instructions described in the manual. The analyzers "Tecan" (Oesterreich), "Pointe-180" ("Scientific", USA) and "Reflotron" (Boehringer Mannheim, BRD) were used with appropriate sets and a fiery spectrophotometer "SF-47".

Immunological parameters were determined by tests as described in the handbook [9] and the previously developed algorithm [8]. The state of the

(C) Popovych I.L., Polovynko I.S., Zajats L.M., Melnyk O.I., 2018 
phagocytic function of neutrophils (microphages) and monocytes (macrophages) is judged by the phagocytic index, microbial (phagocytic) number and the index of killing regarding the museum culture Staphylococcus aureus (ATCC N 25423 F49) [4,5], with the calculation of bactericidal capacity (the number of microbes that are able to neutralize neutrophils or monocytes contained in 1 litre of blood) [4].

Among the parameters of the immunogram the relative amount of blood population of T-cells was determined by M. Jondal's spontaneous rosette test with sheep erythrocytes [6], their theophylline resistant (T-helper) and theophylline sensitive (T-cytotoxic) subpopulations (by S. Limatibul's test of sensitivity rosette to theophylline [10]), the population of B-lymphocytes by C. Bianco's test of complementary rosette of sheep erythrocytes [3]. Natural killers were identified as big containing granules lymphocytes.

After the blood sample, spleen, thymus and adrenal glands were removed and weighed since spleen and thymus did smears for counting splenocytogram and thymocytogram [2,4]. For the latter, as well as for leukocytogram and immunocytogram we calculated entropy [25]. The thickness of glomerular, fascicular, reticular and medullar zones were measured under a microscope in sections of the adrenal glands. $5.5^{\prime \prime}$.

Digital material was processed using the package of softwares "Statistica

Results and discussion. In order to compare different parameters adequately and their ranking, they were listed according to two indices. In this case, the average values of the male indices (M) were used as the base, for which each individual indexes were calculated for each female (F): F/M and (F/M - 1)/Cv, where $\mathrm{Cv}$ is the coefficient of variation of the parameter, common for both sexes. Neuroendocrine parameters, which are significantly different between sexes, are given in Table 1.

Table 1

Neuroendocrine indices of intact males/females and their interrelation

\begin{tabular}{|c|c|c|c|c|}
\hline \multirow[b]{2}{*}{ Variables } & \multicolumn{2}{|c|}{ Actual values } & \multirow{2}{*}{$\begin{array}{l}\text { Females/ } \\
\text { Males }\end{array}$} & \multirow{2}{*}{$\begin{array}{c}(\text { Females/ } \\
\text { Males - 1)/Cv }\end{array}$} \\
\hline & $\begin{array}{c}\text { Males } \\
(n=10)\end{array}$ & $\begin{array}{c}\text { Females } \\
(n=10)\end{array}$ & & \\
\hline $\begin{array}{l}\text { Reticulary Zone Adrenal Cortex, } \\
\mu \mathrm{M}\end{array}$ & $20,0 \pm 1,8$ & $40,8 \pm 2,5$ & $2,04 \pm 0,13^{c}$ & $+4,27 \pm 0,52^{\mathrm{c}}$ \\
\hline Fasciculary Zone Adr Cortex, $\mu \mathrm{M}$ & $218 \pm 11$ & $370 \pm 20$ & $1,70 \pm 0,09^{\mathrm{c}}$ & $+4,15 \pm 0,56^{\mathrm{c}}$ \\
\hline Adrenals Mass, mg & $50 \pm 2$ & $69 \pm 3$ & $1,39 \pm 0,06^{\mathrm{c}}$ & $+3,01 \pm 0,49^{\mathrm{c}}$ \\
\hline Moda HRV, msec & $189 \pm 11$ & $124 \pm 5$ & $0,66 \pm 0,0^{\mathrm{c}}$ & $+2,21 \pm 0,16^{\mathrm{c}}$ \\
\hline $\begin{array}{l}\text { Glomerulary Zone Adrenal Cor- } \\
\text { tex, } \mu \mathrm{M}\end{array}$ & $129 \pm 11$ & $189 \pm 8$ & $1,47 \pm 0,07^{\mathrm{c}}$ & $+2,20 \pm 0,31^{\mathrm{c}}$ \\
\hline Calcitonin Activity as $(\mathrm{Cap} \cdot \mathrm{Pp})^{-0,5}$ & $0,48 \pm 0,02$ & $0,65 \pm 0,05$ & $1,36 \pm 0,11^{\mathrm{b}}$ & $+1,73 \pm 0,55^{b}$ \\
\hline $\begin{array}{l}\text { Parathyrin Activity as }(\mathrm{Cap} \cdot \mathrm{Pu} / \\
\mathrm{Cau} \cdot \mathrm{Pp})^{0,25}\end{array}$ & $2,97 \pm 0,05$ & $3,40 \pm 0,19$ & $1,15 \pm 0,06^{a}$ & $+1,27 \pm 0,55^{\mathrm{a}}$ \\
\hline Parathyrin Activity as $(\mathrm{Cap} \cdot \mathrm{Pu})^{0,5}$ & $1,67 \pm 0,03$ & $2,11 \pm 0,16$ & $1,27 \pm 0,10^{a}$ & $+1,48 \pm 0,54^{\mathrm{a}}$ \\
\hline $\begin{array}{l}\text { Mineralocortic Activ as }(\mathrm{Nap} \cdot \mathrm{Ku} / \\
\mathrm{Kp} \cdot \mathrm{Nau})^{0,25}\end{array}$ & $2,08 \pm 0,12$ & $2,73 \pm 0,25$ & $1,31 \pm 0,12^{a}$ & $+1,33 \pm 0,51^{\mathrm{a}}$ \\
\hline 17-KS urine, $\mathrm{nM} / 100 \mathrm{~g} \cdot$ day & $26 \pm 6$ & $45 \pm 7$ & $1,75 \pm 0,27^{\mathrm{a}}$ & $+1,24 \pm 0,45^{\mathrm{a}}$ \\
\hline $\begin{array}{l}\text { Calcitonin Activity as }(\mathrm{Cau} \cdot \mathrm{Pu} / \\
\mathrm{Cap} \cdot \mathrm{Pp})^{0,25}\end{array}$ & $2,45 \pm 0,04$ & $2,71 \pm 0,12$ & $1,11 \pm 0,05^{a}$ & $+1,09 \pm 0,52^{\mathrm{a}}$ \\
\hline Corticosterone, $\mathrm{nM} / \mathrm{L}$ & $333 \pm 43$ & $466 \pm 57$ & $1,40 \pm 0,17^{\mathrm{a}}$ & $+1,02 \pm 0,43^{\mathrm{a}}$ \\
\hline $\begin{array}{l}\text { Mineralocorticoid Activity as } \\
(\mathrm{Nap} / \mathrm{Kp})^{0,5}\end{array}$ & $6,10 \pm 0,37$ & $5,57 \pm 0,17$ & $0,91 \pm 0,03^{\mathrm{b}}$ & $-0,60 \pm 0,20^{\mathrm{b}}$ \\
\hline Triiodo-thyronine, nM/L & $2,50 \pm 0,17$ & $2,12 \pm 0,18$ & $0,85 \pm 0,07^{\mathrm{a}}$ & $-0,63 \pm 0,30^{a}$ \\
\hline Testosterone, nM/L & $35,1 \pm 4,6$ & $3,93 \pm 0,34$ & $0,11 \pm 0,01^{\mathrm{c}}$ & $-2,57 \pm 0,03^{c}$ \\
\hline
\end{tabular}

Note: $\mathrm{p}<0,05^{\mathrm{a}} ;<0,01^{\mathrm{b}} ;<0,001^{\mathrm{c}}$. 
It was found that the most significant sexual differences were observed in the morpho-functional parameters of the adrenal glands. In particular, the females had the highest androgenic, glucocorticoid and mineralocorticoid activity which was estimated for the thickness of the reticular cortex of the adrenal glands and daily excretion of 17-ketosteroids with urine, the thickness of the fascicular corticoadrenal zone and the plasma corticosterone as well as glomerular zone of the adrenal cortex and levels in plasma and daily urine of sodium and potassium. However, there was higher parathyroid and calcitonin activity in females, measured by levels in plasma and daily urine calcium and phosphate. In addition, females had a lower level of Moda of HRV as an inverse rate of heart rate, which was subjected to the influence of the so-called humoral regulation channel (circulating catecholamines, glucocorticoids, electrolytes, etc.), while the HRV-markers of sympathetic and vagal tonus did not significantly differ, making $53 \pm 7 \%, 56 \pm 5 \%, 45 \pm 8$ and $53 \pm 13 \mathrm{msec}$ respectively. Instead, the plasma level of triiodothyronine in females was $85 \pm 15 \%$ of males, and an order of magnitude lower testosterone level does not need to be commented.

Among the registered immune indices, 11 of them are significantly higher in females (Table 2). First of all, it is the content in the thymocytogram of lymphocytes and lymphoblasts, in the immunocytogram of blood of natural killers and B-lymphocytes, as well as fibroblasts, macrophages and microphages in splenocytogram. In addition, females had higher levels of phagocytosis by monocytes of blood Staph aureus and leukocytosis as well as the entropy of splenocytogram and immunocytogram.

Table 2

Significantly higher immune parameters of intact female rats

\begin{tabular}{|l|c|c|c|c|}
\hline \multirow{2}{*}{ Variables } & \multicolumn{2}{c|}{ Actual values } & Females/ & (Females/ \\
Males & $\begin{array}{c}\text { Males } \\
(\boldsymbol{n}=\mathbf{1 0})\end{array}$ & $\begin{array}{c}\text { Females } \\
(\boldsymbol{n}=\mathbf{1 0})\end{array}$ & Males - 1)/Cv \\
\hline Lymphocytes of Thymus, \% & $55,6 \pm 1,0$ & $70,3 \pm 0,8$ & $1,26 \pm 0,01^{\mathrm{c}}$ & $+5,75 \pm 0,24^{\mathrm{c}}$ \\
\hline NK-Lymphocytes of Blood, \% & $10,4 \pm 0,6$ & $15,0 \pm 0,4$ & $1,45 \pm 0,03^{\mathrm{c}}$ & $+3,53 \pm 0,27^{\mathrm{c}}$ \\
\hline Lymphoblastes of Thymus, \% & $5,50 \pm 0,17$ & $7,40 \pm 0,27$ & $1,35 \pm 0,05^{\mathrm{c}}$ & $+3,29 \pm 0,46^{\mathrm{c}}$ \\
\hline $\begin{array}{l}\text { Microbial Count of Monocytes, } \\
\text { Bact/Phagoc }\end{array}$ & $2,8 \pm 0,1$ & $4,4 \pm 0,5$ & $1,56 \pm 0,16^{\mathrm{b}}$ & $+2,48 \pm 0,73^{\mathrm{c}}$ \\
\hline Fibroblastes of Spleen, \% & $5,90 \pm 0,38$ & $8,20 \pm 0,66$ & $1,39 \pm 0,11^{\mathrm{b}}$ & $+1,69 \pm 0,49^{\mathrm{b}}$ \\
\hline Macrophages of Spleen, \% & $5,50 \pm 0,65$ & $7,90 \pm 0,50$ & $1,44 \pm 0,09^{\mathrm{c}}$ & $+1,51 \pm 0,32^{\mathrm{c}}$ \\
\hline B-Lymphocytes of Blood, \% & $12,7 \pm 0,7$ & $15,4 \pm 0,8$ & $1,21 \pm 0,06^{\mathrm{b}}$ & $+1,27 \pm 0,36^{\mathrm{b}}$ \\
\hline Leukocytes of Blood, 10\%/L & $9,57 \pm 0,54$ & $13,10 \pm 1,76$ & $1,37 \pm 0,17^{\mathrm{c}}$ & $+1,22 \pm 0,58^{\mathrm{a}}$ \\
\hline Entropy of Splenocytogramm $\left(\cdot 10^{3}\right)$ & $721 \pm 9$ & $753 \pm 9$ & $1,04 \pm 0,01^{\mathrm{c}}$ & $+1,17 \pm 0,33^{\mathrm{b}}$ \\
\hline Entropy of Immunocytogramm $\left(\cdot 10^{3}\right)$ & $849 \pm 9$ & $874 \pm 6$ & $1,03 \pm 0,01^{\mathrm{c}}$ & $+1,07 \pm 0,23^{\mathrm{c}}$ \\
\hline Neutrophils of Spleen, \% & $11,5 \pm 0,5$ & $13,0 \pm 0,4$ & $1,13 \pm 0,04^{\mathrm{b}}$ & $+1,06 \pm 0,32^{\mathrm{b}}$ \\
\hline
\end{tabular}

Instead, 10 indicators of immunity in females were significantly lower (Table 3). This is, first and foremost, the activity of phagocytosis of neutrophils/microphages and, to a lesser extent, of monocytes/macrophages, entropy of thymocytograms and the proportion of epithelial cells, endothelial cells and macrophages in it, the proportion of lymphocytes and lymphoblasts in splenocytogram, 0-lymphocytes in immunocytogram as well as the completeness of phagocytosis of neutrophils blood. 

https://doi.org/

Table 3

Significantly lower immune parameters of intact female rats

\begin{tabular}{|c|c|c|c|c|}
\hline \multirow[b]{2}{*}{ Variables } & \multicolumn{2}{|c|}{ Actual values } & \multirow{2}{*}{$\begin{array}{c}\text { Females/ } \\
\text { Males }\end{array}$} & \multirow{2}{*}{$\begin{array}{c}\text { (Females/ } \\
\text { Males - 1)/Cv }\end{array}$} \\
\hline & $\begin{array}{c}\text { Males } \\
(n=10)\end{array}$ & $\begin{array}{c}\text { Females } \\
(n=10)\end{array}$ & & \\
\hline Killing Index of Neutrophils, \% & $54,7 \pm 2,0$ & $50,4 \pm 1,6$ & $0,92 \pm 0,03^{a}$ & $-0,72 \pm 0,27^{\mathrm{a}}$ \\
\hline Lymphoblastes of Spleen, \% & $5,10 \pm 0,38$ & $3,90 \pm 0,38$ & $0,76 \pm 0,07^{\mathrm{b}}$ & $-0,87 \pm 0,27^{b}$ \\
\hline 0-Lymphocytes of Blood, \% & $29,8 \pm 1,5$ & $22,2 \pm 1,9$ & $0,75 \pm 0,07^{\mathrm{b}}$ & $-1,17 \pm 0,30^{b}$ \\
\hline Macrophages of Thymus, \% & $4,70 \pm 0,21$ & $2,70 \pm 0,42$ & $0,57 \pm 0,09^{\mathrm{c}}$ & $-1,33 \pm 0,28^{c}$ \\
\hline Lymphocytes of Spleen, \% & $53,1 \pm 0,9$ & $48,7 \pm 0,9$ & $0,92 \pm 0,02^{\mathrm{c}}$ & $-1,51 \pm 0,30^{c}$ \\
\hline Phagocytose Index of Monocytes, \% & $7,8 \pm 1,1$ & $2,7 \pm 0,2$ & $0,35 \pm 0,03^{\mathrm{c}}$ & $-1,86 \pm 0,08^{\mathrm{c}}$ \\
\hline Endotheliocytes of Thymus, \% & $7,4 \pm 0,4$ & $2,6 \pm 0,3$ & $0,35 \pm 0,04^{\mathrm{c}}$ & $-2,34 \pm 0,15^{\mathrm{c}}$ \\
\hline Epitheliocytes of Thymus, \% & $19,9 \pm 0,7$ & $8,8 \pm 0,6$ & $0,44 \pm 0,03^{\mathrm{c}}$ & $-3,26 \pm 0,18^{c}$ \\
\hline Entropy of Thymocytogramm $\left(\cdot 10^{3}\right)$ & $567 \pm 9$ & $416 \pm 10$ & $0,73 \pm 0,02^{\mathrm{c}}$ & $-4,08 \pm 0,28^{\mathrm{c}}$ \\
\hline Phagocytose Index of Neutrophils, \% & $82,3 \pm 0,7$ & $70,1 \pm 1,0$ & $0,85 \pm 0,01^{\mathrm{c}}$ & $-4,12 \pm 0,35^{\mathrm{c}}$ \\
\hline
\end{tabular}

The described results of the analysis of sexual dimorphism of the neuroendocrine-immune complex of intact rats are visualized in Figures 1 and 2.

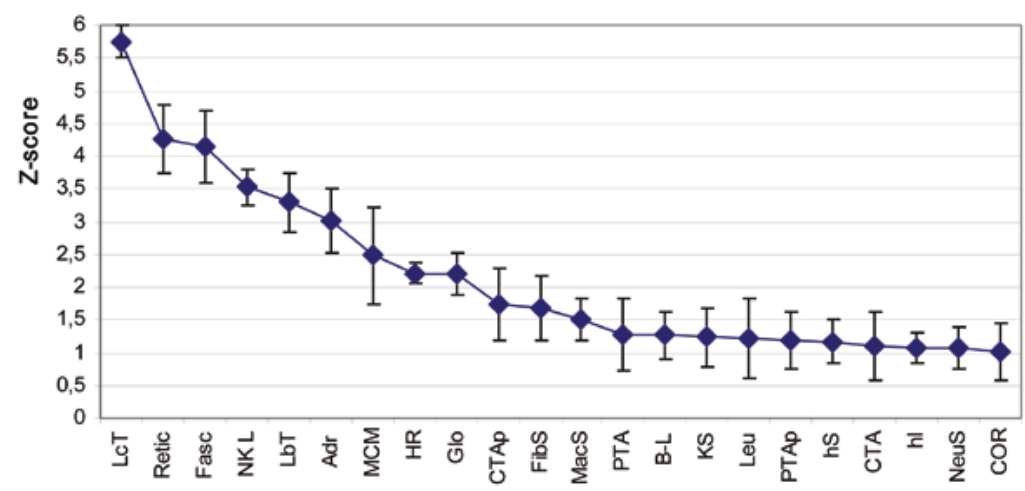

Fig. 1. Significantly larger ranking of Z-scores of neuroendocrine and immune parameters of intact female rats

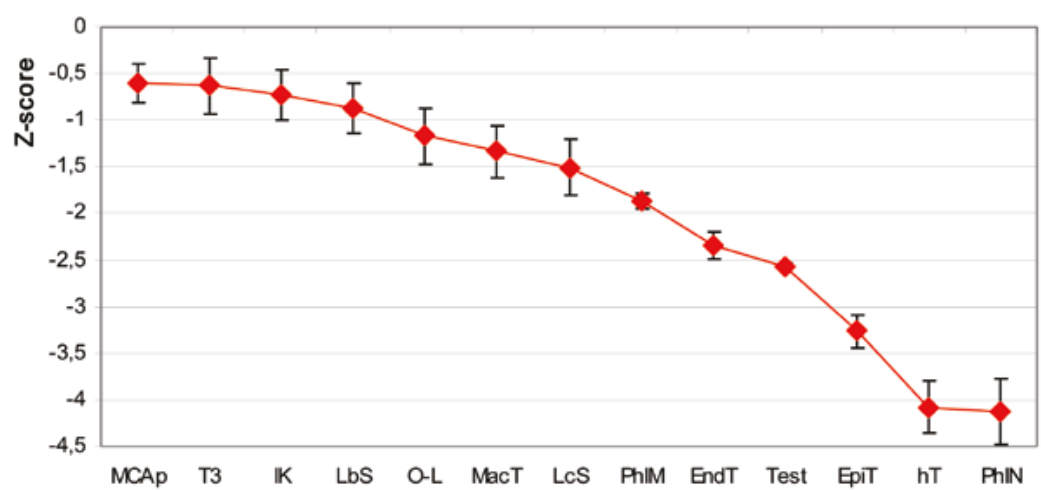

Fig. 2. Ranking of Z-scores of neuroendocrine and immune parameters of intact rats, significantly lower in females

The purpose of the next stage of the analysis was to identify those parameters of the neuroendocrine-immune complex that characterized sexual dimorphism both in normal and after chronic stress. 38 indicators were selected by the method of discriminatory analysis (forward stepwise [7]), including 6 of thymus, 6 of spleen, 13 of blood and 13 of neuroendocrine ones (Table 4). 
The distinguishing information is condensed in three canonical roots. At the same time, the major roots contain its lion's share $(97,9 \%)$, while in the second and third only 1,2 and $0,9 \%$ respectively.

Table 4

\section{Distinguishing Function Analysis Summary}

Step 38, N of variables in model: 38; Grouping: 4 groups

Wilks' Lambda: 0,002; approx. $\mathrm{F}_{(114)}=13 ; p<10^{-6}$

\begin{tabular}{|c|c|c|c|c|c|c|c|c|c|c|}
\hline $\begin{array}{c}\text { Current } \\
\text { Variables } \\
\text { in the model }\end{array}$ & $\begin{array}{l}< \\
\text { in } \\
=1 \\
=1\end{array}$ & 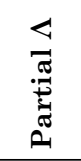 & 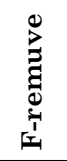 & $\frac{8}{2}$ & 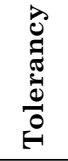 & 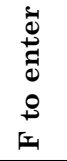 & $\frac{\overrightarrow{0}}{2}$ & $<$ & 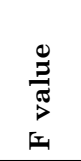 & $\begin{array}{l}\frac{D}{0} \\
\frac{0}{2} \\
\frac{1}{2}\end{array}$ \\
\hline Thymus Mass, mg & ,002 & 883 & 3,1 & ,03 & ,08 & 1,3 & ,27 & ,003 & 15,8 & $10^{-6}$ \\
\hline Thymus Mass Index, \%o & ,002 & ,837 & 4,5 & 01 & ,07 & 1,9 &, 13 & 004 & 18,3 & $10^{-6}$ \\
\hline Epitheliocytes of Thymus, \% &, 002 &, 815 & 5,2 & $10^{-2}$ &, 54 & 190 & $10^{-6}$ &, 157 & 190 & $10^{-6}$ \\
\hline Hassal corpuscles of Thymus, \% &, 002 &, 872 & 3,4 & ,02 & ,34 & 1,7 & ,17 & 004 & 17,2 & $10^{-6}$ \\
\hline Macrophages of Thymus, \% &, 003 & ,742 & 8,0 & $10^{-4}$ & ,34 & 6,2 & $10^{-3}$ &, 021 & 33,6 & $10^{-6}$ \\
\hline Endotheliocytes of Thymus, $\%$ & 003 & 637 & 13 & $10^{-6}$ & ,41 & 19 & $10^{-6}$ & 061 & 60,8 & $10^{-6}$ \\
\hline Spleen Mass, mg & ,002 & ,928 & 1,8 & ,16 & 49 & 1,5 & ,22 & 002 & 13,4 & $10^{-6}$ \\
\hline Reticulocytes of Spleen, \% &, 002 & ,938 & 1,5 & ,21 &, 45 & 1,1 & ,35 &, 002 & 14,0 & $10^{-6}$ \\
\hline Fibroblastes of Spleen, \% & ,002 & 974 & 0,6 & ,61 & ,44 & 1,1 & ,37 & 003 & 15,4 & $10^{-6}$ \\
\hline Neutrophils of Spleen, \% &, 002 & ,949 & 1,2 & ,30 & ,49 & 1,1 & ,37 &, 002 & 13,1 & $10^{-6}$ \\
\hline Eosinophils of Spleen, \% & 003 &, 738 & 8,2 & $10^{-4}$ & ,44 & 2,4 & ,07 & 009 & 21,8 & $10^{-6}$ \\
\hline Plasmocytes of Spleen, \% & 002 & 809 & 5,4 & $10^{-2}$ & ,23 & 3,6 & ,02 & 008 & 21,3 & $10^{-6}$ \\
\hline Phagocytose Ind of Monocyte, \% & 003 &, 615 & 14 & $10^{-6}$ & ,23 & 8,5 & $10^{-4}$ & 038 & 42,8 & $10^{-6}$ \\
\hline Microbial Count Mon, Bac/Phag &, 002 & 950 & 1,2 & ,31 &, 24 & 8,6 & $10^{-4}$ & 025 & 36,0 & $10^{-6}$ \\
\hline Bactericidal Capac Mon, $10^{6} \mathrm{~B} / \mathrm{L}$ & ,002 & ,862 & 3,7 & ,02 & ,36 & 3,3 & ,02 & ,012 & 25,1 & $10^{-6}$ \\
\hline Phagocytose Ind of Neutroph, \% & ,003 & ,681 & 11 & $10^{-5}$ & ,28 & 7,1 & $10^{-3}$ & 031 & 38,3 & $10^{-6}$ \\
\hline Microbial Count Neut, Bac/Phag & 002 & ,798 & 5,8 & $10^{-3}$ & ,37 & 4,0 & ,01 & ,014 & 27,8 & $10^{-6}$ \\
\hline Segmented Neutror & 002 & 848 & 4,1 & ,01 & ,33 & 3,1 & ,03 & 005 & 19,7 & $10^{-6}$ \\
\hline Eosinophils of Blood, \% &, 002 & ,866 & 3,6 & 02 & ,37 & 2,7 & ,05 &, 010 & 22,8 & $10^{-6}$ \\
\hline Basophils of Blood, \% &, 002 & 860 & 3,7 & 01 & ,45 & 1,6 & ,20 & 004 & 17,7 & $10^{-6}$ \\
\hline B-Lymphocytes of Blood, \% &, 002 & 891 & 2,8 & 04 &, 19 & 1,7 & 18 &, 003 & 14,4 & $10^{-6}$ \\
\hline T-cytotoxic Lymphocyt Blood, \% & ,002 & 880 & 3,1 & ,03 &, 15 & 1,6 & ,19 & ,003 & 14,6 & $10^{-6}$ \\
\hline NK-Lymphocytes of Blood, \% & ,002 & ,900 & 2,5 & ,06 & ,38 & 9,9 & $10^{-5}$ & ,047 & 49,4 & $10^{-6}$ \\
\hline 0-Lymphocytes of Blood, \% &, 002 & ,854 & 3,9 & $10^{-2}$ & 09 & 2,8 & ,05 & ,006 & 20,1 & $10^{-6}$ \\
\hline Entropy Immunocytogramm &, 002 &, 858 & 3,8 & ,01 & ,26 & 1,2 & ,32 & 003 & 14,9 & $10^{-6}$ \\
\hline Adrenals Mass, mg &, 002 &, 835 & 4,6 & $10^{-2}$ & ,56 & 5,6 & $10^{-3}$ & 018 & 31,6 & $10^{-6}$ \\
\hline Glomerulary Zone Adr Cortex, $\mu \mathrm{M}$ & ,002 & ,966 & 0,8 & ,49 & ,44 & 2,5 & ,06 &, 011 & 23,8 & $10^{-6}$ \\
\hline MCA as $(\mathrm{Nap} \cdot \mathrm{Ku} / \mathrm{Kp} \cdot \mathrm{Nau})^{0,25}$ & ,002 &, 889 & 2,8 &, 07 &, 44 & 2,4 & ,09 &, 005 & 18,6 & $10^{-6}$ \\
\hline Fasciculary Zone Adr Cortex, $\mu \mathrm{M}$ & ,003 & ,776 & 6,7 & $10^{-3}$ & ,33 & 24 & $10^{-6}$ & ,094 & 79,4 & $10^{-6}$ \\
\hline Corticosterone, $\mathrm{nM} / \mathrm{L}$ &, 002 & ,899 & 2,6 & ,06 &, 46 & 2,3 &, 08 &, 004 & 18,8 & $10^{-6}$ \\
\hline Reticulary Zone Adr. Cortex, $\mu \mathrm{M}$ & ,002 &, 872 & 3,4 & ,02 &, 56 & 2,5 & ,07 & ,005 & 19,2 & $10^{-6}$ \\
\hline Testosterone, nM/L & ,002 & ,929 & 1,8 & ,16 & ,67 & 1,5 & ,23 & ,003 & 16,7 & $10^{-6}$ \\
\hline Thyroxine, nM/L &, 002 & ,892 & 2,8 & ,05 & 29 & 1,6 & ,20 &, 003 & 16,3 & $10^{-6}$ \\
\hline Triiodo-thyronine, nM/L & 002 & ,787 & 6,2 & $10^{-3}$ & ,23 & 3,6 & ,02 &, 006 & 20,7 & $10^{-6}$ \\
\hline Parathyrine activity as $(\mathrm{Cap} / \mathrm{Pp})^{0,5}$ & ,002 & 945 & 1,3 & ,27 & 10 & 3,6 & ,02 &, 013 & 26,4 & $10^{-6}$ \\
\hline PTA as $(\mathrm{Cap} \cdot \mathrm{Pu} / \mathrm{Cau} \cdot \mathrm{Pp})^{0,25}$ & ,002 & ,915 & 2,1 &, 10 & ,09 & 1,2 & ,33 & ,002 & 13,6 & $10^{-6}$ \\
\hline Moda HRV, msec & ,003 &, 759 & 7,3 & $10^{-3}$ & ,12 & 3,7 & ,01 & ,016 & 29,4 & $10^{-6}$ \\
\hline Sympathetic tone as AMo HRV, \% & ,003 & ,754 & 7,5 & $10^{-3}$ & ,13 & 4,5 & ,01 & 007 & 21,0 & $10^{-6}$ \\
\hline
\end{tabular}


The calculation of individual values of discriminant roots was based on the individual values of discriminant variables as well as their raw coefficients and constants, given in Table 5, makes it possible to visualize the localization of each of the 110 rats in the information box of discriminant roots (Fig. 3).

Table 5

Standardized and Raw Coefficients and Constants for Canonical Variables

\begin{tabular}{|c|c|c|c|c|c|c|}
\hline \multirow{2}{*}{$\begin{array}{c}\text { Current } \\
\text { Variables } \\
\text { in the model }\end{array}$} & \multicolumn{3}{|c|}{$\begin{array}{c}\text { Standardized } \\
\text { Coefficients }\end{array}$} & \multicolumn{3}{|c|}{ Raw Coefficients } \\
\hline & $\begin{array}{c}\text { Root } \\
1\end{array}$ & $\begin{array}{c}\text { Root } \\
2\end{array}$ & $\begin{array}{c}\text { Root } \\
3\end{array}$ & $\begin{array}{c}\text { Root } \\
1\end{array}$ & $\begin{array}{c}\text { Root } \\
2\end{array}$ & $\begin{array}{c}\text { Root } \\
3\end{array}$ \\
\hline Thymus Mass, mg &,- 818 & ,238 & $-1,205$ &,- 042 & ,012 &,- 062 \\
\hline Thymus Mass Index, \%o & ,991 &,- 697 & 1,348 & 12,13 & $-8,54$ & 16,49 \\
\hline Epitheliocytes of Thymus, \% &,- 542 & ,281 & 101 &,- 225 & 117 & ,042 \\
\hline Hassal corpuscles of Thymus, \% & ,296 &,- 537 & ,482 & ,438 &,- 796 &, 714 \\
\hline Macrophages of Thymus, \% &,- 003 & $-1,139$ &, 014 &,- 002 &,- 823 & ,010 \\
\hline Endotheliocytes of Thymus, \% &,- 764 &, 720 &,- 040 &,- 601 &, 567 &,- 031 \\
\hline Spleen Mass, mg &, 034 &,- 054 &, 537 &, 000 &,- 000 &, 004 \\
\hline Reticulocytes of Spleen, \% &, 032 &, 187 &,- 480 &, 018 &, 103 &,- 265 \\
\hline Fibroblastes of Spleen, \% & ,095 &, 148 & ,273 & ,056 & ,087 &, 161 \\
\hline Neutrophils of Spleen, \% & ,296 & , 107 &, 144 & , 138 & ,050 & ,067 \\
\hline Eosinophils of Spleen, \% &,- 754 &, 125 & ,216 &,- 836 & , 138 & ,239 \\
\hline Plasmocytes of Spleen, \% &,- 671 &, 674 &, 477 &,- 483 &, 485 &, 343 \\
\hline Phagocytose Index of Monocytes, \% & $-1,057$ & ,974 &,- 138 &,- 609 &, 561 &,- 079 \\
\hline Microbial Count Monocytes, Bacter/Phagocyte &, 011 &,- 585 & , 128 & ,006 &,- 337 &, 074 \\
\hline Bactericidal Capacity of Monocytes, $10^{6}$ Bact/L &,- 386 &, 419 &, 515 &,- 005 &, 006 &, 007 \\
\hline Phagocytose Index of Neutrophils, \% &,- 907 &,- 723 &,- 121 &,- 239 &,- 190 &,- 032 \\
\hline Microbial Count of Neutrophils, Bacter/Phagoc &, 515 &, 216 &, 701 &, 500 &, 210 &, 681 \\
\hline Segmented Neutroph of Blood, \% &,- 028 &,- 809 &,- 374 &,- 005 &,- 142 &,- 066 \\
\hline Eosinophils of Blood, \% &,- 355 &, 185 & ,661 &,- 181 & ,094 & ,336 \\
\hline Basophils of Blood, \% &,- 472 &, 357 &,- 176 & $-1,163$ & ,879 &,- 433 \\
\hline B-Lymphocytes of Blood, \% &,- 548 &, 217 &,- 708 &,- 194 &, 077 &,- 252 \\
\hline T-cytotoxic Lymphocytes of Blood, \% &,- 800 &,- 324 &,- 432 &,- 257 &,- 104 &,- 139 \\
\hline NK-Lymphocytes of Blood, \% &, 278 &, 293 &,- 515 &, 146 &, 154 &,- 271 \\
\hline 0-Lymphocytes of Blood, \% & $-1,131$ & ,493 &,- 466 &,- 172 &, 075 &,- 071 \\
\hline Entropy of Immunocytogramm $\left(\cdot 10^{3}\right)$ & \begin{tabular}{|l|}
, 616 \\
\end{tabular} &, 474 & ,242 & ,022 &, 017 &, 009 \\
\hline Adrenals Mass, mg &, 520 & ,205 & ,042 &, 047 &, 018 &, 004 \\
\hline Glomerulary Zone of Adrenal Cortex, $\mu \mathrm{M}$ &, 053 &, 353 &, 055 &, 002 &, 011 &, 002 \\
\hline $\begin{array}{l}\text { Mineralocorticoid Activity } \\
\text { as }(\mathrm{Nap} \cdot \mathrm{Ku} / \mathrm{Kp} \cdot \mathrm{Nau})^{0,25}\end{array}$ &, 170 & ,900 &,- 145 &, 355 & 1,850 &,- 300 \\
\hline Fasciculary Zone of Adrenal Cortex, $\mu \mathrm{M}$ &, 446 &,- 884 &,- 233 &, 007 &,- 013 &,- 003 \\
\hline Corticosterone, nM/L &,- 392 &,- 272 &, 219 &,- 002 &,- 001 &, 001 \\
\hline Reticulary Zone of Adrenal Cortex, $\mu \mathrm{M}$ & ,451 &,- 213 & ,018 & ,049 &,- 023 & ,002 \\
\hline Testosterone, nM/L &,- 317 &,- 067 &,- 078 &,- 027 &,- 006 &,- 007 \\
\hline Thyroxine, nM/L &,- 587 &,- 114 &,- 192 &,- 037 &,- 007 &,- 012 \\
\hline Triiodo-thyronine, nM/L &,- 866 &,- 250 &,- 528 & $-1,738$ &,- 502 & $-1,060$ \\
\hline Parathyrine Activity as $(\mathrm{Cap} / \mathrm{Pp})^{0,5}$ &, 173 &, 904 &,- 147 &, 354 & 1,852 &,- 301 \\
\hline Parathyrine Activity as $(\mathrm{Cap} \cdot \mathrm{Pu} / \mathrm{Cau} \cdot \mathrm{Pp})^{0,25}$ &, 172 &,- 789 & 1,048 &, 386 & $-1,777$ & 2,358 \\
\hline Moda HRV, msec & $-1,202$ &,- 556 &,- 768 &,- 042 &,- 019 &,- 027 \\
\hline \multirow[t]{2}{*}{ Sympathetic tone as AMo HRV, \% } & $-1,363$ &, 077 &,- 178 &,- 059 &, 003 &,- 008 \\
\hline & \multicolumn{3}{|c|}{ Constants } & 22,16 & 4,04 & $-1,92$ \\
\hline
\end{tabular}



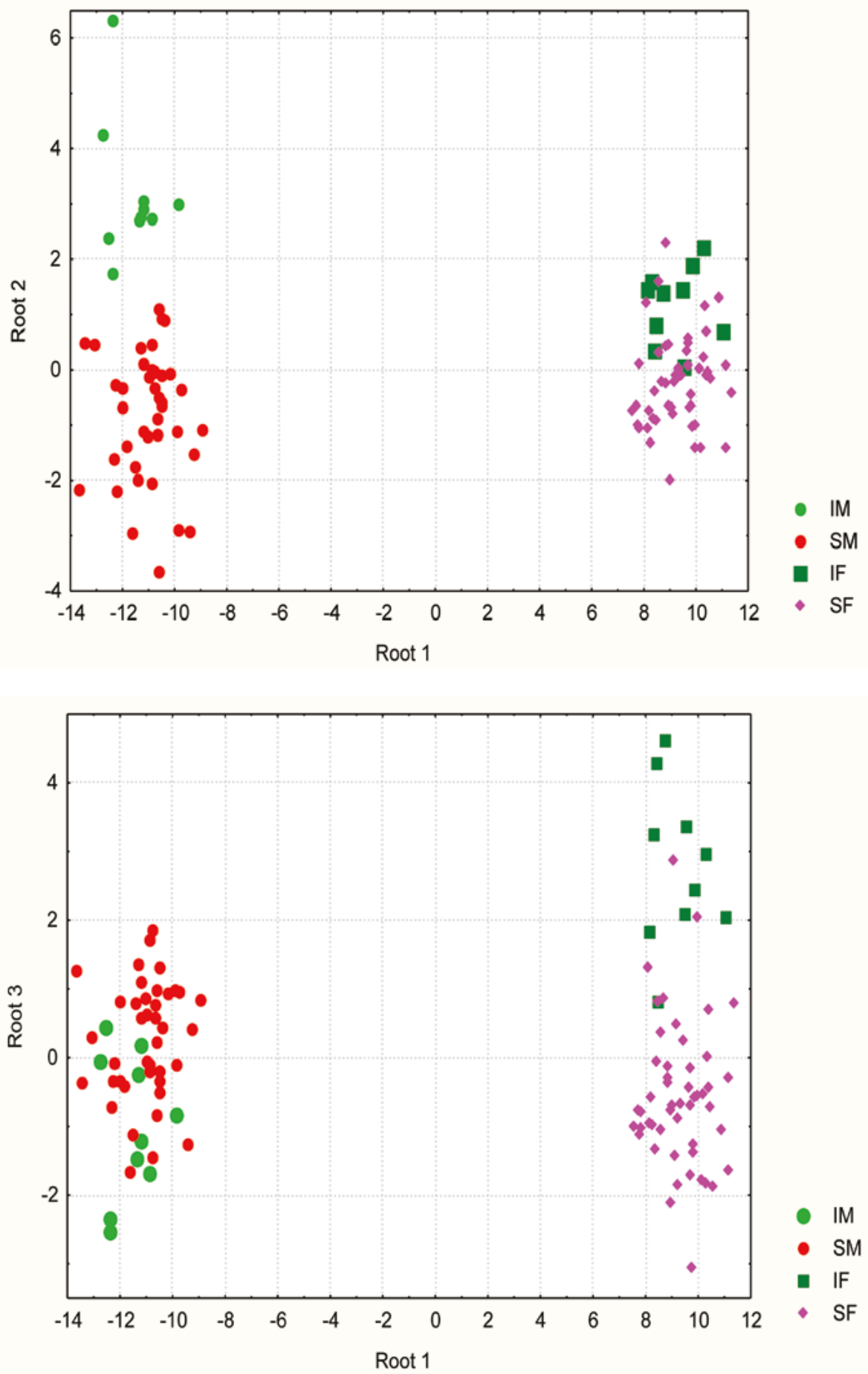

Fig. 3. Individual sizes of canonical discriminant roots of intact (I) and stressed (S) males $(\mathrm{M})$ and females $(\mathrm{F})$ rats

After calculating the average values (centroids) of discriminant roots (Table 6), it is possible to visualize the localization of four groups of animals on their planes (Fig. 4). 
Table 6

Means of Canonical Variables and Chi-Square Tests with Successive Roots Removed

\begin{tabular}{|l|c|c|c|}
\hline & Root 1 & Root 2 & Root 3 \\
\hline Intact Males $(n=10)$ & $-11,6$ & 3,2 & $-1,0$ \\
\hline Stressed Males $(n=40)$ & $-11,0$ & $-0,8$ & 0,2 \\
\hline Intact Females $(n=10)$ & 9,2 & 1,2 & 2,8 \\
\hline Stressed Females $(n=50)$ & 9,3 & $-0,2$ & $-0,6$ \\
\hline Eigenvalues & 107,5 & 1,4 & 1,0 \\
\hline Canonical R & 0,995 & 0,760 & 0,705 \\
\hline Wilks' Lambda & 0,002 & 0,212 & 0,503 \\
\hline Chi-Square & 549 & 136 & 61 \\
\hline Degree freedom & 114 & 74 & 36 \\
\hline p-level & $10-6$ & $10-4$ &, 006 \\
\hline
\end{tabular}
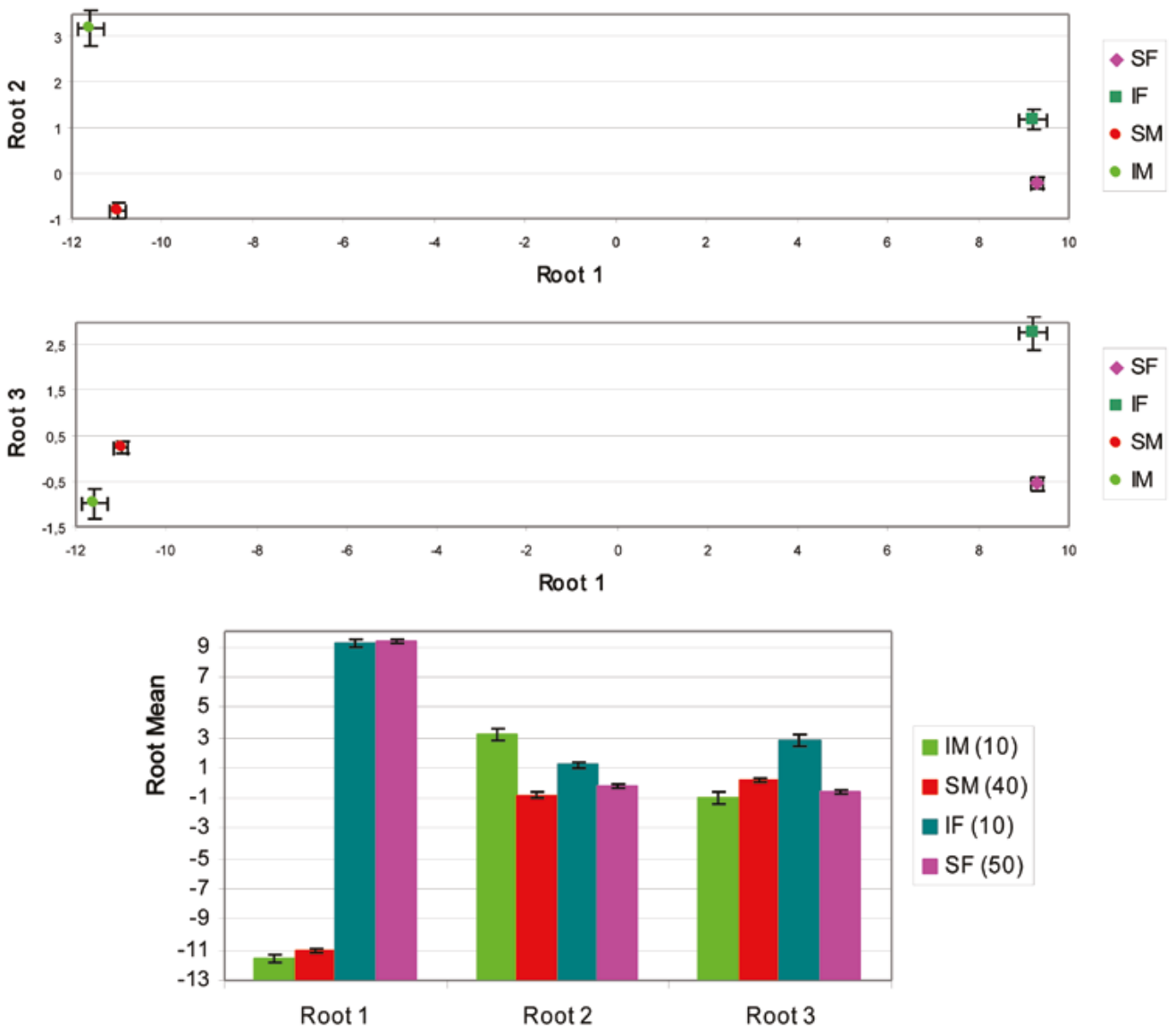

Fig. 4. Average values $(\mathrm{M} \pm \mathrm{m})$ of canonical discriminant roots of intact (I) and stressed (S) males $(\mathrm{M})$ and females $(\mathrm{F})$ rats

The drastic delineation along the axis of the first root of males and females reflects the values of the parameters of the neuroendocrine-immune complex significantly more in males, which are correlated with the root negatively, as well as significantly smaller than females, are correlated with the root of the parameters positively. At the same time, for each sex, these parameters do not differ significantly in intact and stressed animals, that is, they are stress-resistant (Table 7). 
Factor Structure Matrix. Correlations of Variables-Canonical Roots (within-groups correlations)

\begin{tabular}{|c|c|c|c|c|c|c|c|}
\hline $\begin{array}{l}\text { Variables currently } \\
\text { in the model }\end{array}$ & $\begin{array}{c}\text { Root } \\
1\end{array}$ & $\begin{array}{c}\text { Root } \\
2\end{array}$ & $\begin{array}{c}\text { Root } \\
3\end{array}$ & $\begin{array}{c}\text { Intact } \\
\text { Males } \\
(10)\end{array}$ & $\begin{array}{c}\text { Stressed } \\
\text { Males } \\
(40) \\
\end{array}$ & $\begin{array}{c}\text { Intact } \\
\text { Females } \\
(10)\end{array}$ & $\begin{array}{c}\text { Stressed } \\
\text { Females } \\
(50)\end{array}$ \\
\hline Epitheliocytes of Thymus, \% &,- 223 &,- 105 &,- 047 & $19,9 \pm 0,7$ & $20,6 \pm 0,4$ & $8,8 \pm 0,6$ & $9,4 \pm 0,3$ \\
\hline Phagocytose Ind of Neutroph., \% &,- 161 &, 016 &, 046 & $82,3 \pm 0,7$ & $81,9 \pm 0,6$ & $70,1 \pm 1,0$ & $69,4 \pm 0,5$ \\
\hline Endotheliocytes of Thymus, \% &,- 143 & 201 &,- 109 & $7,40 \pm 0,43$ & $6,15 \pm 0,25$ & $2,60 \pm 0,31$ & $2,65 \pm 0,13$ \\
\hline Testosterone, nM/L &,- 137 &,- 124 &,- 007 & $35,1 \pm 4,6$ & $40,0 \pm 2,7$ & $3,93 \pm 0,34$ & $4,87 \pm 0,32$ \\
\hline Macrophages of Thymu &,- 097 &,- 257 &, 018 & $4,70 \pm 0,21$ & $6,13 \pm 0,28$ & $2,70 \pm 0,42$ & $3,08 \pm 0,16$ \\
\hline Moda HRV, msec &,- 096 &, 172 &, 022 & $189 \pm 11$ & $168 \pm 6$ & $124 \pm 5$ & $114 \pm 3$ \\
\hline 0-Lymphocytes of Blood, \% &,- 086 &,- 096 &, 118 & $29,8 \pm 1,5$ & $33,0 \pm 0,9$ & $22,2 \pm 1,9$ & $\pm 1,1$ \\
\hline Triiodo-thyronine, nM/L &,- 037 &,- 099 &,- 056 & $2,50 \pm 0,17$ & $2,65 \pm 0,10$ & $2,12 \pm 0,18$ & $2,28 \pm 0,05$ \\
\hline Plasmocytes of Spleen, \% &,- 036 &,- 003 & , 138 & $2,80 \pm 0,47$ & $3,03 \pm 0,24$ & $2,50 \pm 0,50$ & $1,86 \pm 0,17$ \\
\hline Eosinophils of Spleen, \% &,- 033 &,- 093 &, 049 & $1,80 \pm 0,25$ & $2,18 \pm 0,16$ & $1,52 \pm 0,33$ & $1,49 \pm 0,11$ \\
\hline NK-Lymphocytes of Blood, \% &, 160 & ,098 &,- 183 & $10,4 \pm 0,6$ & $9,4 \pm 0,2$ & $15,0 \pm 0,4$ & $16,0 \pm 0,3$ \\
\hline Fasciculary Zone Adr Cortex, $\mu \mathrm{M}$ &, 118 &,- 130 &,- 119 & $217 \pm 11$ & & $370 \pm 20$ & $417 \pm$ \\
\hline Glomerulary Zone Adr Cort, $\mu \mathrm{M}$ &, 104 &, 125 &,- 017 & $129 \pm 11$ & $13 \pm 3$ & $189 \pm 8$ & $188 \pm 6$ \\
\hline $\mathrm{MCA}$ as $(\mathrm{Nap} \cdot \mathrm{Ku} / \mathrm{Kp} \cdot \mathrm{Nau})^{0,25}$ &, 100 &, 120 &,- 015 & $2,08 \pm 0,12$ & $2,18 \pm 0,14$ & $2,73 \pm$ & $3,05 \pm 0,14$ \\
\hline Reticulary Zone Adr. Cortex, $\mu \mathrm{M}$ &, 104 &,- 096 &,- 024 & $20,0 \pm 1,8$ & $23,5 \pm 1,1$ & $40,7 \pm 2,5$ & $43,6 \pm 1,6$ \\
\hline Adrenals Mass, mg &, 092 &, 015 &,- 040 & $49,6 \pm 1,8$ & $49,0 \pm 1,6$ & $69,0 \pm 3,2$ & $70,5 \pm 1,8$ \\
\hline Entropy Im &, 070 & ,078 &,- 101 & & & & $881 \pm 3$ \\
\hline B-Lym &, 056 &,- 002 &,- 054 & $12,7 \pm 0,7$ & 0,4 & $15,4 \pm 0,8$ & $0 \pm 0,4$ \\
\hline Fibroblas &, 055 &,- 006 & & $5,90 \pm 0,38$ & $6,10 \pm 0,28$ & $8,20 \pm 0,66$ & $7,94 \pm 0,23$ \\
\hline Neu &, 050 &, 096 & & & & $13,0 \pm 0,4$ & $13,0 \pm 0,3$ \\
\hline T-cytotoxic Lymph &, 038 &, 033 &,- 105 & 0,9 & & $15,1 \pm 0,9$ & $16,1 \pm 0,4$ \\
\hline Microbial Count Mon, Bact/Phag &, 034 &,- 121 &,- 012 & $2,8 \pm 0,1$ & & 0,5 & $4,7 \pm 0,3$ \\
\hline Reticulocytes of Spleen, \% &, 034 &, 057 &,- 156 & $14,3 \pm 0,6$ & $13,6 \pm 0,3$ & $14,3 \pm 0,6$ & $15,1 \pm 0,3$ \\
\hline Phagocytose Ind of Monocytes, \% &,- 090 &, 254 &,- 149 & $7,8 \pm 1,1$ & $5,6 \pm 0,3$ & $2,7 \pm 0,2$ & $2,9 \pm 0,1$ \\
\hline Basophils of Bloc &, 018 & ,062 &,- 123 & $0,37 \pm 0,14$ & $0,21 \pm 0,05$ & $0,28 \pm 0,12$ & $0,41 \pm 0,07$ \\
\hline Segment Neutrophils of Blood, \% &,- 002 &, 021 &,- 036 & $28,1 \pm 1,7$ & $27,4 \pm 0,7$ & $26,9 \pm 2,2$ & $27,4 \pm 0,9$ \\
\hline Sympathet tone as AMo HRV, \% &,- 003 &,- 144 &,- 028 & & & & $63 \pm 3$ \\
\hline Thymus Mass, mg &,- 024 &,- 145 &, 031 & & & & \\
\hline Thymus Mass Index, \%o &,- 026 &,- 130 & & $0,29 \pm 0,02$ & $4 \pm 0,01$ & $0,28 \pm 0,04$ & $0,29 \pm 0,01$ \\
\hline Hassal corpuscles of Thymus, \% &, 005 &,- 102 &,- 080 & $1,70 \pm 0,27$ & $1,91 \pm 0,14$ & $1,70 \pm 0,17$ & $1,98 \pm 0,06$ \\
\hline Corticosterone, nM/L & 011 &,- 077 &, 081 & $333 \pm 43$ & $411 \pm 34$ & $466 \pm 57$ & $433 \pm 27$ \\
\hline $\mathrm{PTA}$ as $(\mathrm{Cap} \cdot \mathrm{Pu} / \mathrm{Cau} \cdot \mathrm{Pp})^{0,25}$ &, 024 &, 130 & ,316 & $2,97 \pm 0,05$ & $2,92 \pm 0,03$ & $3,61 \pm 0,22$ & $3,05 \pm 0,08$ \\
\hline Parathyrine activity as $(\mathrm{Cap} / \mathrm{Pp})^{0,5}$ &, 027 & 131 & ,309 & $1,67 \pm 0,06$ & $1,60 \pm 0,03$ & $2,38 \pm 0,25$ & $1,78 \pm 0,09$ \\
\hline Microbial Count Neutr, Bact/Phag &,- 007 &, 115 &, 196 & $8,1 \pm 0,1$ & $7,9 \pm 0,1$ & $8,5 \pm 0,5$ & $7,7 \pm 0,2$ \\
\hline Bactericidal Capac Mon, $10^{6} \mathrm{~B} / \mathrm{L}$ &,- 017 &,- 024 & 191 & $81 \pm 14$ & $103 \pm 10$ & $110 \pm 52$ & $67 \pm 7$ \\
\hline Eosinophils of Blood, \% &,- 002 &,- 010 &, 115 & $3,50 \pm 0,62$ & $3,85 \pm 0,32$ & $4,30 \pm 0,68$ & $3,57 \pm 0,27$ \\
\hline Spleen Mass, mg &, 011 &, 031 & , 108 & $730 \pm 50$ & $732 \pm 19$ & $816 \pm 83$ & $756 \pm 20$ \\
\hline Thyroxine, nM/L &,- 007 &,- 014 &, 097 & $60 \pm 6$ & $62 \pm 3$ & $64 \pm 5$ & $59 \pm 2$ \\
\hline Discriminant Properties, \% & 97,9 & 1,2 & 0,9 & & & & \\
\hline
\end{tabular}

Instead, intact and stressed males are clearly delimited along the axis of the second root, which reflects the post-stress decrease in the phagocytic activity of monocytes in the blood and the content of basophils and segmental neutrophils in it, on the one hand, and the increase in the mass of thymus and the content of Hassall's cells in it, and the increase of the sympathetic tone and corticosterone - on the other hand. Post-stress changes of the listed parameters in females in general are insignificant. Significant differences between intact 
and stressed females are reflected along the third root axis, which contains the information on stress induced lowering levels of parathyrin and thyroxin, the intensity of Staph aureus phagocytosis by microphages and bactericidal capacity of blood macrophages, its content of eosinophils as well as the mass of the spleen. Instead, post-stress changes of the listed parameters in males are generally insignificant.

The obvious differences between the four groups of rats are documented by calculating the squares of Mahalanobis distances (Table 8).

Squared Mahalanobis Distances, F-values $(\mathrm{df}=39)$ and p-levels

\begin{tabular}{|c|c|c|c|c|}
\hline Groups & IM & SM & IF & SF \\
\hline $\begin{array}{l}\text { Intact Male } \\
(n=10)\end{array}$ & 0 & 19 & 468 & 465 \\
\hline $\begin{array}{l}\text { Stressed Male } \\
(n=40)\end{array}$ & $\begin{array}{l}2,3 \\
10^{-3}\end{array}$ & 0 & 436 & 430 \\
\hline $\begin{array}{l}\text { Intact Female } \\
(n=10)\end{array}$ & $\begin{array}{c}36 \\
10^{-6}\end{array}$ & $\begin{array}{c}55 \\
10^{-6}\end{array}$ & 0 & 14 \\
\hline $\begin{array}{l}\text { Stressed Female } \\
(n=50)\end{array}$ & $\begin{array}{c}61 \\
10^{-6}\end{array}$ & $\begin{array}{l}160 \\
10^{-6}\end{array}$ & $\begin{array}{l}1,8 \\
10^{-2}\end{array}$ & 0 \\
\hline
\end{tabular}

Consequently, the discrepancies between neuroendocrine-immune complexes of intact and stressed rats of both sexes are explained exhaustively by 38 parameters. The information contained in these metrics can be condensed in three recognition roots. Selected parameters can be used to identify each of the four animal groups by means of calculating the classifying functions by their coefficients and constants (Table 9) and the individual values of discriminant parameters.

Table 9

Coefficients and Constants for Classifying Functions

\begin{tabular}{|c|c|c|c|c|}
\hline $\begin{array}{l}\text { Current Variables } \\
\text { in the model }\end{array}$ & $\begin{array}{c}\text { Intact } \\
\text { Male }\end{array}$ & $\begin{array}{l}\text { Stressed } \\
\text { Male }\end{array}$ & $\begin{array}{l}\text { Intact } \\
\text { Female }\end{array}$ & $\begin{array}{c}\text { Stressed } \\
\text { Female }\end{array}$ \\
\hline 1 & 2 & 3 & 4 & 5 \\
\hline Thymus Mass, mg & 1,41 & 1,26 & 27 & 46 \\
\hline Thymus Mass Index, \%o & -435 & -374 & -104 & -146 \\
\hline Epitheliocytes of Thymus, \% & 9,86 & 9,32 & 5,10 & 4,78 \\
\hline Hassal corpuscles of Thymus, \% & $-25,09$ & $-20,78$ & $-11,69$ & $-12,91$ \\
\hline Macrophages of Thymus, \% & 3,37 & 6,68 & 5,01 & 6,13 \\
\hline Endotheliocytes of Thymus, \% & 10,99 & 8,34 & $-2,78$ & $-3,52$ \\
\hline Spleen Mass, mg & 10 & 11 & ,12 & ,11 \\
\hline Reticulocytes of Spleen, \% & 15,76 & 15,04 & 14,94 & 15,68 \\
\hline Fibroblastes of Spleen, \% & 10,18 & 10,06 & 11,77 & 11,12 \\
\hline Neutrophils of Spleen, \% & 3,99 & 3,95 & 7,00 & 6,72 \\
\hline Eosinophils of Spleen, \% & 41,94 & 41,21 & 25,17 & 24,11 \\
\hline Plasmocytes of Spleen, \% & 35,26 & 33,47 & 25,54 & 23,67 \\
\hline Phagocytose Index of Monocytes, \% & 19,93 & 17,25 & 5,85 & 5,27 \\
\hline Microbial Count Monocytes, Bacter/Phagocyte & 35,04 & 36,49 & 36,12 & 36,35 \\
\hline Bactericidal Capacity of Monocytes, $10^{6}$ Bact/L &,- 028 &,- 046 &,- 127 &,- 160 \\
\hline Phagocytose Index of Neutrophils, \% & 21,37 & 21,97 & 16,67 & 17,03 \\
\hline Microbial Count of Neutrophils, Bacter/Phagoc & $-6,59$ & $-6,33$ & 5,94 & 3,42 \\
\hline Segmented Neutrophils of Blood, \% & $-1,26$ &,- 77 & $-1,33$ &,- 91 \\
\hline Eosinophils of Blood, \% & $-8,21$ & $-8,28$ & $-10,90$ & $-12,17$ \\
\hline Basophils of Blood, \% & $-22,83$ & $-27,53$ & $-50,40$ & $-50,29$ \\
\hline
\end{tabular}


Continuation of table 9

\begin{tabular}{|l|c|c|c|c|}
\hline \multicolumn{1}{|c|}{$\mathbf{1}$} & $\mathbf{2}$ & $\mathbf{3}$ & $\mathbf{4}$ & $\mathbf{5}$ \\
\hline B-Lymphocytes of Blood, \% & 10,75 & 10,03 & 5,60 & 6,32 \\
\hline T-cytotoxic Lymphocytes of Blood, \% & 3,02 & 3,13 & $-2,64$ & $-2,05$ \\
\hline NK-Lymphocytes of Blood, \% & $-3,75$ & $-4,61$ & $-2,03$ & $-1,33$ \\
\hline 0-Lymphocytes of Blood, \% & 6,97 & 6,48 & 2,97 & 3,08 \\
\hline Entropy of Immunocytogramm $\left(\cdot 10^{3}\right)$ & 2,140 & 2,094 & 2,602 & 2,550 \\
\hline Adrenals Mass, mg &,- 39 &,- 43 &, 56 &, 53 \\
\hline Glomerulary Zone of Adrenal Cortex, $\mu \mathrm{M}$ &, 55 &, 51 &, 57 &, 55 \\
\hline $\begin{array}{l}\text { Mineralocorticoid Activity as }(\mathrm{Nap} \cdot \mathrm{Ku} / \\
\text { Kp·Nau) }\end{array}$ & 123 & 133 & 143 & 138 \\
\hline Fasciculary Zone of Adrenal Cortex, $\mu \mathrm{M}$ &, 14 &, 19 &, 29 &, 32 \\
\hline Corticosterone, nM/L &, 13 &, 14 &, 10 &, 09 \\
\hline Reticulary Zone of Adrenal Cortex, $\mu \mathrm{M}$ & $-1,78$ & $-1,66$ &,- 70 &,- 67 \\
\hline Testosterone, nM/L &,- 24 &,- 24 &,- 81 &,- 79 \\
\hline Thyroxine, nM/L & 2,51 & 2,51 & 1,72 & 1,77 \\
\hline Triiodo-thyronine, nM/L & 170,4 & 170,1 & 131,2 & 135,3 \\
\hline Parathyrine Activity as $(\mathrm{Cap} / \mathrm{Pp})^{0,5}$ & $-100,8$ & $-108,4$ & $-98,3$ & $-99,9$ \\
\hline Parathyrine Activity as $(\mathrm{Cap} \cdot \mathrm{Pu} / \mathrm{Cau} \cdot \mathrm{Pp})^{0,25}$ & 122,9 & 133,1 & 143,3 & 138,0 \\
\hline Moda HRV, msec & 3,53 & 3,55 & 2,60 & 2,71 \\
\hline Sympathetic tone as AMo HRV, \% & 4,01 & 3,95 & 2,74 & 2,75 \\
\hline Constants & -3293 & -3287 & -2822 & -2814 \\
\hline
\end{tabular}

In this case, the accuracy of the classification is $100 \%$.

Conclusion. The data obtained by us indicate the need to take into account the sexual differences in the reactions of the neuroendocrine-immune complex to stress when testing the stress limiting means.

\section{REFERENCES}

1. Baevskiy RM, Kirillov OI, Kletskin SZ. Mathematical Analysis of Changes in Heart Rate by Stress [in Russian]. Moskva: Nauka. 1984. 221 p. 2. Bazarnova MA. Cytology investigation punctates spleen. In: Guide to practical training in clinical laboratory diagnostics [in Russian]. Kyiv: Vyshcha shkola. 1988: 263-4. 3. Bianco C. Population of lymphocytes bearing a membrane receptor for antigen-antibody complex. J Exp Med. 1970; 134(4): 702-20. 4. Bilas VR, Popovych $I L$. Role of microflora and organic substances of water Naftussya in its modulating influence on neuroendocrine-immune complex and metabolism [in Ukrainian]. Medical Hydrology and Rehabilitation. 2009; 7(1): 68-102. 5. Douglas SD, Quie PG. Investigation of Phagocytes in Disease. Churchil. 1981. 110 p. 6. Jondal M, Holm G, Wigzell H. Surface markers on human T and B lymphocytes. I. A large population of lymphocytes forming nonimmune rosettes with sheep red blood cells. J Exp Med. 1972; 136(2): 207-15. 7. Klecka WR. Discriminant Analysis [trans. from English in Russian] (Seventh Printing, 1986). In: Factor, Discriminant and Cluster Analysis. Moskva: Finansy i Statistika. 1989: 78-138. 8. Kozyavkina OV, Kozyavkina NV, Gozhenko OA, Gozhenko AI, Barylyak LG., Popouych IL. Bioactive Water Naftussya and Neuro-EndocrineImmune Complex [in Ukrainian]. Kyiv: UNESCO-SOCIO. 2015. 349 p. 9. Lapovets' LYe, Lutsyk $B D$. Handbook of Laboratory Immunology [in Ukrainian]. Lviv. 2002. 173 p. 10. Limatibul S, Shore A, Dosch HM, Gelfand $E W$. Theophylline modulation of E-rosette formation: an indicator of T-cell maturation. Clin Exp Immunol. 1978; 33(3): 503-13. 11. Polovynko IS. Integrated quantitative estimation of neuro-endocrine manifestations of chronic stress in female rats. Experimental and Clinical Physiology and Biochemistry. 2017; 3(79): 5-10. 12. Polovynko IS, Popovych IL. Immunotropic traits in responses to chronic stress rats males [in Ukrainian]. In: VV Podvysotskiy XI reading. Bulletin Materials Science Conference (Odesa, 24-25 May, 2012). Odessa: Ukrainian Research Institute of Transport Medicine, 2012: 109-10. 13. Polovynko IS, Popovych $I L$. Variety immunotropic responses to chronic stress and neuroendocrine accompaniment of male rats [in Ukrainian]. In: Mater. V scientific-practical conference "Issues of pathology in conditions of extreme factors action on the body" (Ternopil, November 1-2, 2012). Achievements of Clinical and Experimental Medicine. 2012; 2(17): 200. 14. Polovynko IS, Zajats LM, Popovych $I L$. Neuroendocrine-immune relationships in males rats in conditions of chronic stress [in Ukrainian]. In: Mater. VI scientific-practical conference "Issues of pathology in conditions of extreme factors action on the body" (Ternopil, October 31-November 1, 2013). Achievements of Clinical and Experimental Medicine. 2013; 2(19): 274. 15. Polovynko IS, Zajats LM, Popouych AI, 
Popovych IL. Integral quantification of neuroendocrine and immune responses to chronic stress in male rats [in Ukrainian]. In: Pathophysiology and Pharmacy: ways of integration: Abstracts VII National Congress pathophysiologists Ukraine with international participation (5-7 October 2016). Kharkiv: NPhU, 2016: 182. 16. Polovynko IS, Zayats LM, Zukow W, Popovych IL. Neuroendocrine-immune relationships by chronic stress at male rats. Journal of Health Sciences. 2013; 3(12): 365-74. 17. Polovynko IS, Zajats LM, Zukow W, Yanchij RI, Popovych IL. Quantitative evaluation of integrated neuro-endocrine and immune responses to chronic stress rats male. Journal of Education, Health and Sport. 2016; 6(8): 154-66. 18. Polovynko IS, Zukow W. Variety of immune responses to chronic stress in rats male. Journal of Education, Health and Sport. 2016; 6(12): 843-56. 19. Popovych IL. Functional interactions between neuroendocrine-immune complex in males rats [in Ukrainian]. Achievements of Clinical and Experimental Medicine. 2008; 2(9): 80-7. 20. Popovych IL. The concept of neuroendocrine-immune complex (Review) [in Russian]. Medical Hydrology and Rehabilitation. 2009; 7(3): 9-18. 21. Sternberg EM. Neural regulation of innate immunity: a coordinated nonspecific host response to pathogens. Nat Rev Immunol. 2006; 6(4): 318-28. 22. Thayer JF, Sternberg EM. Neural aspects of immunomodulation: Focus on the vagus nerve. Brain Behav Immun. 2010; 24(8): 1223-8. 23. Tracey KJ. Reflex control of immunity. Nat Rev Immunol. 2009; 9(6): 418-28. 24. Uchakin PN, Uchakina ON, Tobin BV, Ershov FI. Neuroendocrine immunomodulation [in Russian]. Vestnik Ross AMN. 2007; 9: 26-32. 25. Yushkovs'ka OG. Using information theory to study adaptive responses in the body athletes [in Ukrainian]. Medical Rehabilitation, Kurortology, Physiotherapy. 2001; 1(25): 40-3. 26. Zajats LM, Polovynko IS, Zukow W. Features neuro-endocrine support diversity of immune responses to chronic stress in male rats. Journal of Education, Health and Sport. 2017; 7(3): 97-105. 27. Zajats LM, Polovynko IS, Zukow W, Yanchij RI, Mysakovets' OG, Mel'nyk OI, Hrytsak YaL. Neuroendocrine-immune relatioships in rats females. Journal of Education, Health and Sport. 2017; 7(10): 59-78.

Стаття надійшла до редколегії 31.08.2018

\title{
RESEARCH ARTICLE
}

\section{Sexual Dimorphism of the Neuroendocrine-Immune Complex and its Reactions to Chronic Stress in Rats}

\author{
I.L. POPOVYCH ${ }^{1}$, I.S. POLOVYNKO ${ }^{2}$, L.M. ZAJATS ${ }^{2}$, O.I. MELNYK ${ }^{3}$
}

${ }^{1}$ Department of Immunophysiology, O. Bohomolets Institute of Physiology, Kyiv, Ukraine

${ }^{2}$ Department of Pathophysiology, Ivano-Frankivsk National Medical University, Ivano-Frankivsk, Ukraine

${ }^{3}$ Department of Physiology, Danylo Halytskyi Lviv National Medical University, Lviv, Ukraine

E-mail: i.popovych@biph.kiev.ua

E-mail: patfisiology@ifnmu.edu.ua

Introduction. If the sexual differences in endocrine parameters are well understood, then research of immune parameters in this aspect remains relevant.

Materials and methods. The experiment was performed on 50 males and 60 females of the Wistar line rats. Parameters of HRV, blood and daily urine levels of hormones and electrolytes as well as parameters of leukocytogram, immunocytogram, thymocytogram and splenocytogram were determined.

Results. It was found that the most significant sexual differences were observed in the morpho-functional parameters of the adrenal glands. Intact females had the highest androgenic, glucocorticoid and mineralocorticoid activity in particular, as well as parathyrin and calcitonin activity. The HRV-markers of sympathetic and vagal tones were not significantly different. Instead, the plasma level of triiodothyronine in females was $85 \%$ of males. Among the recorded immune indices, 11 of them were significantly higher in females. First of all, it was the content in the thymocytogram of lymphocytes and lymphoblasts, in the immunocytogram of blood natural killers and B-lymphocytes as well as fibroblasts, macrophages and microphages in splenocytogram. Instead, the 10 indicators of immunity in females were significantly lower. This was, first of all, the activity of phagocytosis of microphages and macrophages. The discrepancies between neuroendocrine-immune complexes of intact and stressed rats of both sexes were exhaustively explained by 38 parameters including 6 of thymus, 6 of spleen, 13 of blood and 13 of neuroendocrine ones. The information contained in these metrics can be condensed in three recognition roots. Intact and stressed males were clearly delimited along the axis of the second root which reflected the post-stress decrease in the phagocytic activity of monocytes in the blood and the content of basophils and segmental neutrophils in it, on the one hand and the increase in the 
mass of thymus and the content of Hassall's cells in it, and the increase of the sympathetic tone and corticosterone - on the other hand. Post-stress changes of the listed parameters in general were insignificant in females. Significant differences between intact and stressed females were reflected along the third root axis which contains the information on stress induced lowering levels of parathyrin and thyroxin, the intensity of Staph aureus phagocytosis by microphages and bactericidal capacity of blood macrophages, its content of eosinophils as well as the mass of the spleen. Instead, post-stress changes in the listed parameters were generally insignificant in males.

Conclusions. The obtained data indicate the need to take into account the sexual differences in the reactions of the neuroendocrine-immune complex to stress when testing the stress limiting means.

Key words: sexual difference, neuroendocrine-immune complex, stress, immunological parameters, immunogram, neuroendocrine indices. 